\title{
A Three-Dimensional Model of the Effective Electromechanical Impedance for an Embedded PZT Transducer
}

\author{
Chunyuan Zuo, Xin Feng, and Jing Zhou \\ Faculty of Infrastructure Engineering, Dalian University of Technology, Dalian 116024, China \\ Correspondence should be addressed to Xin Feng; fengxin@dlut.edu.cn
}

Received 15 October 2013; Accepted 4 December 2013

Academic Editor: Ting-Hua Yi

Copyright (c) 2013 Chunyuan Zuo et al. This is an open access article distributed under the Creative Commons Attribution License, which permits unrestricted use, distribution, and reproduction in any medium, provided the original work is properly cited.

\begin{abstract}
A three-dimensional model of the effective electromechanical impedance for an embedded PZT transducer is proposed by considering the interaction between a PZT patch and a host structure. By introducing an effective mechanical impedance, the coupled electromechanical admittance formulations are derived using the piezoelectric constitutive equations. Then, a modified methodology for monitoring structure changes using an electromechanical impedance (EMI) technique is proposed. In the proposed method, the changes in the host structure are monitored by using the "active" part associated with the structural mechanical impedance, which is extracted from the measured raw admittance signatures. The strength gain of a concrete beam with embedded PZT transducers during the curing age was monitored with the proposed methodology. The experimental results demonstrate that the use of the "active" part is more sensitive as opposed to the raw admittance signatures for structural health monitoring (SHM).
\end{abstract}

\section{Introduction}

In general, a typical structural health monitoring (SHM) system includes three major components: a sensor system, a data processing system (including data acquisition, transmission, and storage), and a health evaluation system. The sensor system is the key component in the SHM system $[1,2]$. The advent of commercially available smart piezoelectric materials has provided new opportunities for SHM. The key characteristic of PZT materials is the utilization of the converse piezoelectric effect to actuate the structure in addition to the direct piezoelectric effect to sense structural deformation $[3,4]$. By the direct and converse piezoelectric effects of PZT materials, the electromechanical impedance (EMI) technique simultaneously employs piezoelectric ceramic (PZT) patches as both sensors and actuators.

In recent years, the EMI technique has gained wide attention and emerged as a favorable tool for structural damage detection. This technique can diagnose several types of structures, including bolted connections $[5,6]$, cable-stayed bridges [7], aerospace structures [8], reinforced concrete structures $[9,10]$, and pipeline structures [11]. The basic concept of this approach is to use high-frequency excitations to monitor the local area of the host structure for changes in the structural mechanical impedance caused by damage [12]. In the EMI technique, a small-size PZT patch is bonded to or embedded into the monitored structure and excited by an alternating voltage sweep signal. The PZT patch transfers the vibrations to the host structure, which is deformed and produces a local dynamic response. Simultaneously, the response of the host structure is transferred back to the PZT patch. Because of the electromechanical coupling property of PZT materials, the structural mechanical impedance of the host structure can be determined by the electrical impedance of the PZT patch. By monitoring the variations of the measured electrical impedance, the change in the structural mechanical impedance induced by structural damage can be detected. The advantages of EMI-based approaches include but are not limited to a lightweight and inexpensive sensor, high sensitivity to incipient damage for high-frequency actuating, and immunity to ambient or far-field loads.

The interaction between the PZT patch and the host structure is the key issue for sensor design and data interpretation in EMI-based methods. During the last two decades, 


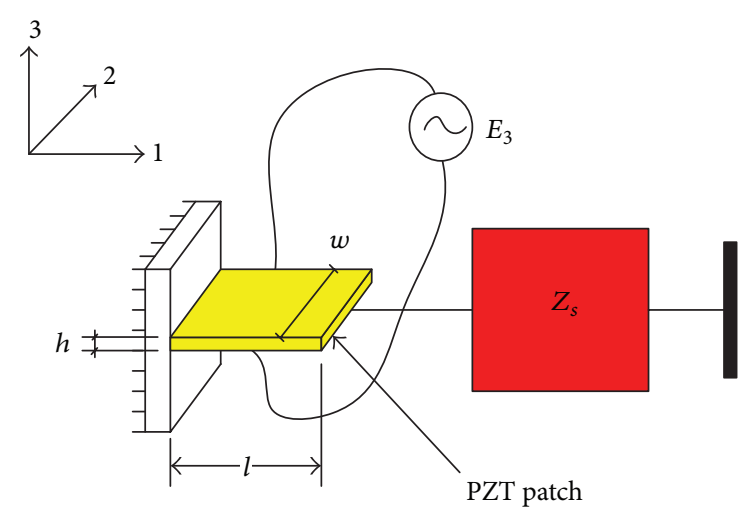

FIGURE 1: Liang's 1D impedance model [13].

several modeling approaches have been proposed for describing the PZT-structure interaction. Among them, two wellknown approaches are the static approach and the impedance approach. The former one developed by Crawley and de Luis [16] uses a statically determined equivalent force and moment as the amplitude of the forcing function. It is assumed that the mechanical interaction between the bonded actuator and the host structure occurs at the ends of the actuator in the form of concentrated forces. This approach is simple and easy to use but it often leads to misleading results. More importantly, the static approach does not correctly capture the physical essence of the PZT-structure interaction. In order to improve the accuracy, Liang et al. [13] presented the impedance approach instead of the static approach. This approach modeled the PZT patch connected to the host structure represented by the mechanical impedance $Z_{s}$, as shown in Figure 1. The multidimensional effects associated with PZT vibrations are ignored in Liang's model. Zhou et al. [14] extended the one-dimensional (1D) model to a generic two-dimensional (2D) PZT-structure model by considering the coupled vibration in the plane. This model replaced the structural impedance with a matrix consisting of the direct impedances $Z_{x x}$ and $Z_{y y}$ and the cross-impedances $Z_{x y}$ and $Z_{y x}$, as shown in Figure 2. Experimental difficulties prevent the direct application of Zhou's model for extraction of the structural mechanical impedance. To overcome this shortcoming, a new concept of "effective impedance" was introduced in the effective impedance model of Bhalla and Soh [15]. In this model, the PZT-structure mechanical interaction extends all over the finite-sized PZT patch, as shown in Figure 3. This model condenses the two-directional physical coupling between the PZT patch and the host structure into a single impedance term, which can be determined from the measured admittance signatures. Hence, the model bridges the gap between Liang's 1D model and Zhou's 2D model.

Conventionally, the impedance models are usually considered as $1 \mathrm{D}$ or $2 \mathrm{D}$, and the coupling between the extensional and longitudinal vibrations is neglected. To satisfy the requirements of the $1 \mathrm{D}$ model, the thickness of the PZT patch must be much smaller than its radius (length and width). However, the PZT patch has finite dimensions of thickness and radius (length and width) in practical applications,

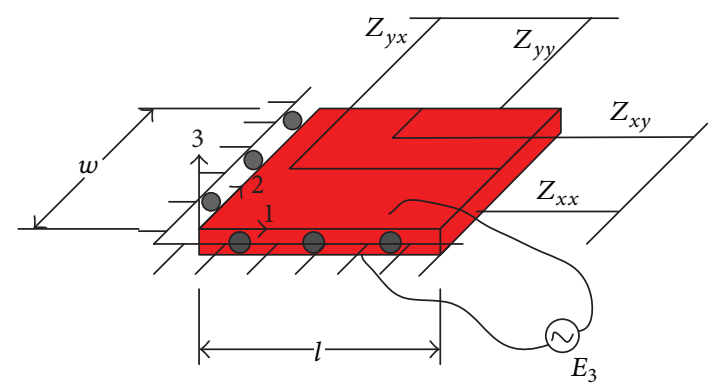

FIGURE 2: Zhou's 2D impedance model [14].

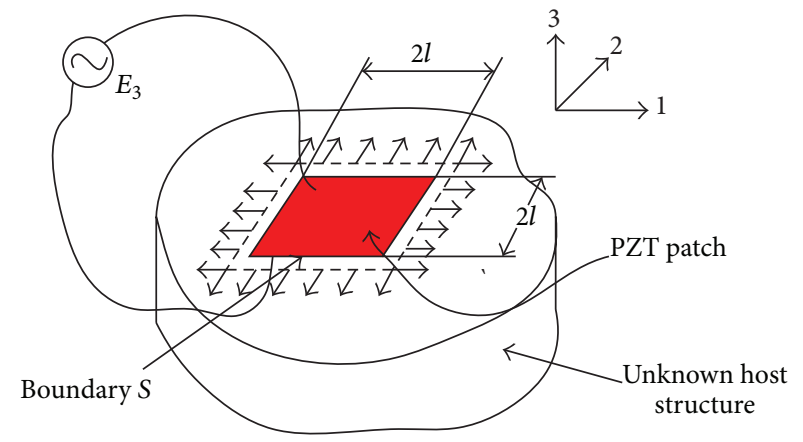

Figure 3: 2D effective impedance model of Bhalla and Soh [15].

and both the extensional and longitudinal vibrations are important [17]. Hence, there is a need for the development of a three-dimensional (3D) PZT-structure interaction model, which considers the coupling between the extensional and longitudinal vibrations. The study presented here aims to model the PZT-structure interaction considering the $3 \mathrm{D}$ coupled vibration effect. In this paper, a 3D effective electromechanical impedance model for an embedded PZT plate is proposed by adopting the "effective impedance" concept. The new model describes the extensional actuations along the radial direction and longitudinal actuation along the thickness direction of the PZT patch. The advantage of the $3 \mathrm{D}$ effective impedance model is that it can only capture the "active part" associated with the structural mechanical impedance by filtering the PZT contribution. Then, the inherent properties of the monitored structure can be detected with a higher accuracy than with the raw admittance signal only. To validate the effectiveness of the proposed method, a concrete beam with embedded PZT transducers during the curing age was measured to monitor the strength gain.

\section{Theoretical Background}

In a linear vibration system, the steady-state response $x(t)$, that is, displacement, velocity, or acceleration, is caused by the harmonic force $F(t) . x(t)$ and $F(t)$ have the same frequency, and $x(t)$ lags behind $F(t)$ by a phase angle $\phi$. The structural mechanical impedance $Z$ is defined as the ratio of the harmonic excitation to its steady-state response. Conventionally, the velocity impedance is regarded as the 
mechanical impedance. Therefore, the structural mechanical impedance is expressed as

$$
Z=\frac{F}{\dot{u}}=\frac{F_{o} e^{j \omega t}}{\dot{u}_{o} e^{j(\omega t-\phi)}}=\frac{F_{o}}{\dot{u}_{o}} e^{j \phi},
$$

where $F_{o}$ is the amplitude of the excitation force, $\omega$ is the circular frequency of the excitation force, $\dot{u}_{o}$ is the amplitude of the velocity, and $\phi$ is the phase angle of the velocity response.

The existing models are based on the definition that the structural mechanical impedance refers to a particular point, and the point considered is the PZT end point. However, the PZT-structure interaction is not restricted at the PZT end point, and it extends all over the PZT patch. The force transmission between the PZT patch and the host structure occurs along the entire boundary of the PZT patch [15]. Ignoring the effect of the PZT vibrations in the thickness direction and assuming the frequency range of interest to be much lower than the dominant modes of thickness vibration, a new concept of "effective mechanical impedance" was defined by Bhalla and Soh [15]. However, the embedded PZT patch has both the extensional and longitudinal vibrations in practical applications. Hence, the formulations of the "effective mechanical impedance" of $3 \mathrm{D}$ model need to be established.

We consider a circular PZT patch embedded into an unknown host structure, as shown in Figure 4, subjected to an electric field. The interaction of the PZT patch with the host structure is represented in the form of the boundary traction $f$, and the PZT-structure interaction force is assumed along the entire boundary of the PZT patch. Further, the PZT patch is assumed to be extremely small as compared to the host structure, and it has negligible mass and stiffness. Hence, the PZT patch can be regarded as an effective drive point. In addition, the impedance of the effective drive point is defined as the effective mechanical impedance.

The "effective mechanical impedance" of the PZT patch is defined as

$$
Z_{a, \mathrm{eff}}=\frac{\oint_{S} f \cdot \widehat{n} d S}{\dot{u}_{\mathrm{eff}}}=\frac{F_{\mathrm{eff}}}{\dot{u}_{\mathrm{eff}}},
$$

where $F_{\text {eff }}$ is the effective force; $\widehat{n}$ is the unit vector normal to the boundary; $u_{\text {eff }}=\delta V / A_{o}$ is defined as effective displacement of the PZT patch, where $\delta V$ is the change in volume of the PZT patch; and $A_{o}$ is the surface area of the PZT patch. Differentiation of the effective displacement with respect to time yields the effective velocity $\dot{u}_{\text {eff. The }}$ "effective mechanical impedance" of the host structure can be defined based on applying the force on the surface of the host structure, along the boundary of the location of the embedded PZT patch.

\section{Three-Dimensional Electromechanical Impedance Models}

The existing impedance models are usually considered to be $1 \mathrm{D}$ or $2 \mathrm{D}$ models, and they neglect the longitudinal vibration

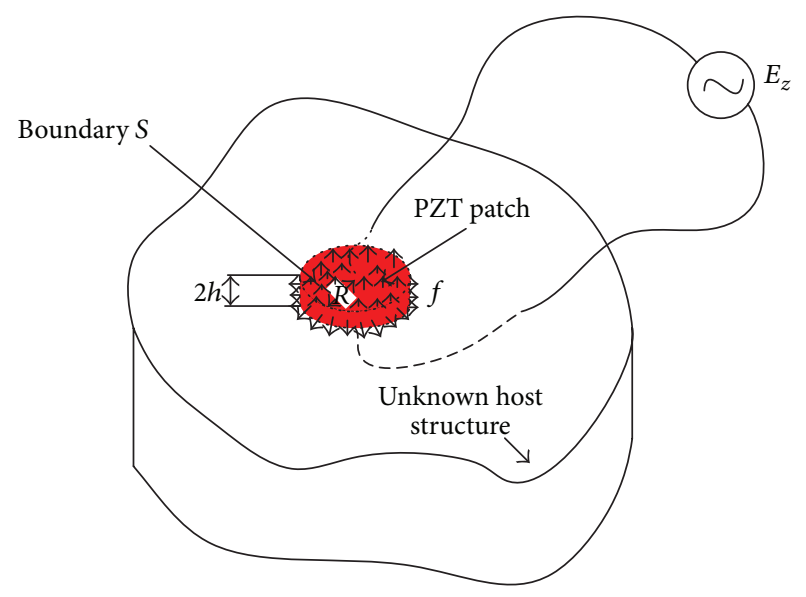

Figure 4: 3D effective impedance model.

of the PZT patch. Hence, there is a need for the development of a 3D PZT-structure interaction model that considers both the extensional and longitudinal vibrations of the PZT patch. By applying the concept of "effective mechanical impedance," a 3D effective impedance model is proposed. The features of the proposed impedance model are that there are no restrictions of the PZT dimensions, and the model more accurately represents the actual situation.

3.1. 3D Electromechanical Admittance Formulation by the Effective Impedance. A circular PZT patch of radius $R$ and thickness $2 h$ is shown in Figure 5. The polarization direction is along the $Z$ axis, and the external exciting electric field $E_{Z}$ is also applied along the $Z$-direction and parallel to the polarization direction. Then, the vibration of the PZT patch can be regarded as a symmetrical vibration, and the shearing effect can be ignored. Owing to the symmetrical vibration, it is sufficient to use $1 / 8$ of the PZT patch and host structure. Because of the mechanical and piezoelectric anisotropy of the PZT patch, the formulations $S_{11}^{E}=1 / \overline{Y^{E}}$, $S_{12}^{E}=-v_{12} S_{11}^{E}=-v_{12} / \overline{Y^{E}}, S_{13}^{E}=-v_{13} S_{11}^{E}=-v_{13} / \overline{Y^{E}}$, and $S_{33}^{E}=$ $\nu_{33} S_{11}^{E}=v_{33} / \overline{Y^{E}}$ are obtained. In cylindrical coordinates, the constitutive relations of the PZT patch are expressed as [18]

$$
\begin{gathered}
D_{z}=\overline{\varepsilon_{33}^{T}} E_{z}+d_{31}\left(T_{r r}+T_{\theta \theta}\right)+d_{33} T_{z z}, \\
S_{r r}=\frac{T_{r r}}{\overline{Y^{E}}}-\frac{\nu_{12} T_{\theta \theta}}{\overline{Y^{E}}}-\frac{\nu_{13} T_{z z}}{\overline{Y^{E}}}+d_{31} E_{z}, \\
S_{\theta \theta}=-\frac{\nu_{12} T_{r r}}{\overline{Y^{E}}}+\frac{T_{\theta \theta}}{\overline{Y^{E}}}-\frac{\nu_{13} T_{z z}}{\overline{Y^{E}}}+d_{31} E_{z}, \\
S_{z z}=-\frac{\nu_{13} T_{r r}}{\overline{Y^{E}}}-\frac{\nu_{13} T_{\theta \theta}}{\overline{Y^{E}}}+\frac{\nu_{33} T_{z z}}{\overline{Y^{E}}}+d_{33} E_{z},
\end{gathered}
$$

where $T_{r r}, T_{\theta \theta}$, and $T_{z z}$ are, respectively, the radial, tangential, and axial stress; $S_{r r}, S_{\theta \theta}$, and $S_{z z}$ are, respectively, the radial, tangential, and axial strain; $S_{i j}^{E}$ is the elastic compliance constant in the short-circuited condition; $\overline{Y^{E}}$ is the complex Young's modulus of elasticity at a constant electric field; 


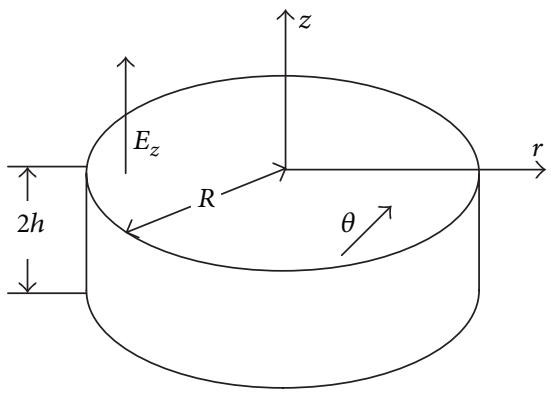

(a)

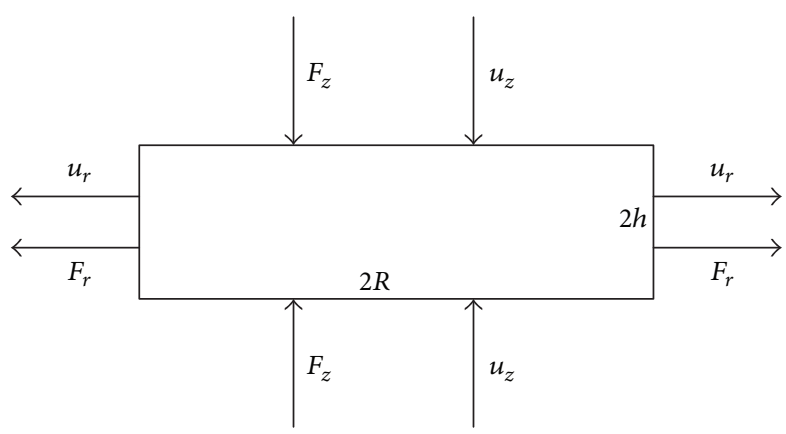

(b)

FIgUre 5: (a) Geometry of the circular PZT patch and (b) stresses and displacements on the PZT patch.

$v_{12}, v_{13}$, and $v_{33}$ are Poisson's ratios; $d_{31}$ and $d_{33}$ are the piezoelectric strain coefficients; $E_{z}$ is the electric field; $D_{z}$ is the electric displacement; and $\frac{z}{\varepsilon_{33}^{T}}$ is the complex electric permittivity at a constant stress.

We let $n=T_{z z} /\left(T_{r r}+T_{\theta \theta}\right) . n$ is defined as the mechanical coupling coefficient between different vibrational modes [17]. By algebraic manipulation, we obtain

$$
\begin{gathered}
T_{r r}+T_{\theta \theta}=\frac{\overline{Y^{E}}}{\lambda}\left[S_{r r}+S_{\theta \theta}+S_{z z}-\left(2 d_{31}+d_{33}\right) E_{z}\right], \\
T_{z z}=\frac{n \overline{Y^{E}}}{\lambda}\left[S_{r r}+S_{\theta \theta}+S_{z z}-\left(2 d_{31}+d_{33}\right) E_{z}\right],
\end{gathered}
$$

where $\lambda=1-v_{12}-v_{13}+n\left(v_{33}-2 v_{13}\right)$. Then, the effective force of the PZT-structure interaction is given by

$$
\begin{aligned}
F & =\oint_{S} f \cdot \widehat{n} d S=\left(T_{r r}+T_{\theta \theta}\right) \cdot \frac{2 \pi R h}{4}+T_{Z Z} \cdot \frac{\pi R^{2}}{4} \\
& =\frac{\pi R(2 h+n R)}{4 \lambda}\left[S_{r r}+S_{\theta \theta}+S_{z z}-\left(2 d_{31}+d_{33}\right) E_{Z}\right] .
\end{aligned}
$$

In the short-circuited condition, the effective force is expressed as

$$
F_{\text {short-circuited }}=\frac{\pi R(2 h+n R) \overline{Y^{E}}}{4 \lambda}\left(S_{r r}+S_{\theta \theta}+S_{z z}\right) .
$$

As derived in $[18,19]$, the displacements of the PZT patch in cylindrical coordinates are provided by

$$
\begin{gathered}
u_{r}=A_{1} J_{1}\left(\kappa_{1} r\right) e^{j \omega t}, \\
u_{\theta}=0, \\
u_{z}=A_{2} \sin \left(\kappa_{2} z\right) e^{j \omega t},
\end{gathered}
$$

where the wave number of the radial direction $\kappa_{1}=$ $\omega \sqrt{\left(2 \rho \lambda\left(1+v_{12}\right)\right) /\left(\left(1+v_{12}+\lambda\right) \overline{Y^{E}}\right)}$ and the wave number of the longitudinal direction $\kappa_{2}=\omega \sqrt{\rho \lambda / n \overline{Y^{E}}} . A_{1}$ and $A_{2}$ are constants determined by the boundary conditions. $J_{1}(x)$ is the Bessel function of the first kind and first order, and $J_{1}^{\prime}(x)=$
$J_{0}(x)-(1 / x) J_{1}(x)$. By differentiating (7) with respect to time, the velocities are obtained as follows:

$$
\begin{gathered}
\dot{u}_{r}=\frac{\partial u_{r}}{\partial t}=A_{1} j \omega J_{1}\left(\kappa_{1} x\right) e^{j \omega t}, \\
\dot{u}_{\theta}=\frac{\partial u_{\theta}}{\partial t}=0, \\
\dot{u}_{z}=\frac{\partial u_{z}}{\partial t}=A_{2} j \omega \sin \left(\kappa_{2} z\right) e^{j \omega t} .
\end{gathered}
$$

The strains are obtained by differentiating (7) with respect to the coordinate axes:

$$
\begin{gathered}
S_{r r}=\frac{\partial u_{r}}{\partial r}=A_{1}\left[\kappa_{1} J_{0}\left(\kappa_{1} r\right)-\frac{1}{r} J_{1}\left(\kappa_{1} r\right)\right] e^{j \omega t}, \\
S_{\theta \theta}=\frac{u_{r}}{r}=\frac{A_{1}}{r} J_{1}\left(\kappa_{1} r\right) e^{j \omega t}, \\
S_{z z}=\frac{\partial u_{z}}{\partial z}=A_{2} \kappa_{2} \cos \left(\kappa_{2} z\right) e^{j \omega t} .
\end{gathered}
$$

Under the multimode vibration and free boundary conditions, the frequency equations for the PZT patch in a coupled vibration are obtained as follows:

$$
\begin{gathered}
\cot \kappa_{2} \cdot 2 h=0 \\
\kappa_{1} R J_{0}\left(\kappa_{1} R\right)=\left(1-\nu_{12}\right) J_{1}\left(\kappa_{1} R\right) .
\end{gathered}
$$

Then, the following relations are obtained by solving (8) and (9):

$$
\begin{gathered}
\kappa_{2} \cdot 2 h=(2 m-1) \frac{\pi}{2}, \quad(m=1,2,3, \ldots), \\
\kappa_{1} R=R(n), \quad(n=1,2,3, \ldots),
\end{gathered}
$$

where $R(n)$ is the root for (9), and it is a function of $v_{12}$. By substituting $\kappa_{1}=\omega \sqrt{\left(2 \rho \lambda\left(1+v_{12}\right)\right) /\left(\left(1+\nu_{12}+\lambda\right) \overline{Y^{E}}\right)}, \kappa_{2}=$ $\omega \sqrt{\rho \lambda / n \overline{Y^{E}}}$, and $\lambda=1-v_{12}-v_{13}+n\left(\nu_{33}-2 v_{13}\right)$ and solving, $n$ is obtained as follows:

$$
n=\frac{2-v_{13}}{\left(1+v_{12}\right) C^{\prime}-\left(v_{33}-2 v_{13}\right)},
$$

where $C^{\prime}=\left((2 i-1)^{2} \pi^{2}\right) /\left(8 R^{2}(j)\right) \cdot\left(R^{2} / h^{2}\right)$. 
Based on the definition of the effective displacement in (1), the displacement at the boundaries of $1 / 8$ of the PZT patch is expressed as

$$
u_{\mathrm{eff}}=\frac{\delta V}{A_{o}} \approx \frac{2 h u_{r o}-R u_{z o}}{2 h+R} .
$$

Differentiating with respect to time, the effective velocity is obtained:

$$
\dot{u}_{\mathrm{eff}} \approx \frac{2 h \dot{u}_{r o}-R \dot{u}_{z o}}{2 h+R} .
$$

To simplify the calculation, we assume that

$$
\frac{u_{z o}}{h}=-v_{13} \cdot \frac{u_{r o}}{R} .
$$

Substituting (7), the following formulations are derived:

$$
\frac{A_{1}}{A_{2}}=-\frac{R \sin \left(\kappa_{2} h\right)}{v_{13} h J_{1}\left(\kappa_{1} R\right)}, \quad \frac{A_{2}}{A_{1}}=-\frac{\nu_{13} h J_{1}\left(\kappa_{1} R\right)}{R \sin \left(\kappa_{2} h\right)} .
$$

Substituting (6), (8), (9), and (14) into (2), the effective impedance of the PZT patch is expressed as

$$
\begin{aligned}
Z_{a, \mathrm{eff}}= & \frac{\pi(2 h+R)(2 h+n R) \overline{Y^{E}}}{4 \lambda\left(2+\nu_{13}\right) h j \omega} \\
& \cdot\left[\frac{\kappa_{1} R J_{0}\left(\kappa_{1} R\right)}{J_{1}\left(\kappa_{1} R\right)}-v_{13} \frac{\kappa_{2} h}{\tan \left(\kappa_{2} h\right)}\right] .
\end{aligned}
$$

The effective force $F$ is related to the effective impedance of the host structure by

$$
F=\oint_{S} f \cdot \widehat{n} d S=-Z_{s, \text { eff }} \cdot \dot{u}_{\text {eff }}
$$

By substituting (8), (9), and (18) into (2), with $E_{z}=\left(V_{o} /\right.$ 2h) $e^{j \omega t}$, the constants $A_{1}$ and $A_{2}$ are obtained:

$$
\begin{aligned}
& A_{1}=\left(\alpha R V_{0} Z_{a, \mathrm{eff}}\right) \\
& \times\left(2 h \cdot J _ { 1 } ( \kappa _ { 1 } R ) \left[\frac{\kappa_{1} R J_{0}\left(\kappa_{1} R\right)}{J_{1}\left(\kappa_{1} R\right)}-v_{13}\right.\right. \\
& A_{2}=\left.\left.\left(-v_{13} \alpha V_{0} Z_{a, \mathrm{eff}}\right) \quad \frac{\kappa_{2} h}{\tan \left(\kappa_{2} h\right)}\right]\left(Z_{s, \mathrm{eff}}+Z_{a, \mathrm{eff}}\right)\right)^{-1}, \\
& \times\left(2 \operatorname { s i n } ( \kappa _ { 2 } h ) \left[\frac{\kappa_{1} R J_{0}\left(\kappa_{1} R\right)}{J_{1}\left(\kappa_{1} R\right)}-v_{13}\right.\right. \\
&\left.\left.\cdot \frac{\kappa_{2} h}{\tan \left(\kappa_{2} h\right)}\right]\left(Z_{s, \mathrm{eff}}+Z_{a, \mathrm{eff}}\right)\right)^{-1} .
\end{aligned}
$$

The instantaneous electric current is expressed as

$$
\bar{I}=\frac{d Q(t)}{d t}=\iint_{A} \dot{D}_{Z} d A=j \omega \iint_{A} D_{Z(Z=h)} d A
$$

Substituting (4), (9), and (19) into (3), the electromechanical admittance of the 3D effective electromechanical impedance model is derived as

$$
\begin{aligned}
\bar{Y}= & \overline{\bar{V}}=G+B j \\
= & \frac{\pi R^{2} j \omega}{2 h} \\
\times & {\left[\overline{\varepsilon_{33}^{T}}-\frac{\alpha \beta \overline{Y^{E}}}{\lambda}\right.} \\
& +\left(\left(\frac{\alpha \beta \overline{Y^{E}}}{\lambda} \cdot\left(2-v_{13} \cdot \frac{\kappa_{2} h}{\tan \left(\kappa_{2} h\right)}\right) \cdot Z_{a, \text { eff }}\right)\right. \\
& \times\left(\left[\frac{\kappa_{1} R J_{0}\left(\kappa_{1} R\right)}{J_{1}\left(\kappa_{1} R\right)}-v_{13} \cdot \frac{\kappa_{2} h}{\tan \left(\kappa_{2} h\right)}\right]\right. \\
& \left.\left.\left.\quad \times\left(Z_{s, \text { eff }}+Z_{a, \text { eff }}\right)\right)^{-1}\right)\right],
\end{aligned}
$$

where $\alpha=2 d_{31}+d_{33}$ and $\beta=d_{31}+n d_{33} ; \bar{V}=V_{o} e^{j \omega t}$ is the instantaneous voltage across the PZT patch.

This formulation contains $Z_{a \text {,eff }}$ and the effective structural mechanical impedance $Z_{s \text {,eff. }} Z_{a \text {,eff }}$ is constant for certain material parameters. The variations in $Z_{s, \text { eff }}$ caused by a change in the inherent properties of the host structure directly influence the electromechanical impedance. Hence, the variations in the inherent properties can be monitored by the measured electromechanical impedance.

3.2. Decomposition of the Coupled Electromechanical Admittance Signatures. In Section 3.1, the complex electromechanical admittance of the proposed impedance model has been derived. By rearranging the various terms in (21), it can be separated into two parts as

$$
\begin{aligned}
\bar{Y}= & \underbrace{\frac{\pi R^{2} j \omega}{2 h} \cdot\left[\overline{\varepsilon_{33}^{T}}-\frac{\alpha \beta \overline{Y^{E}}}{\lambda}\right]}_{\text {Part I }} \\
& +\underbrace{\frac{\pi R^{2} j \omega}{2 h} \cdot \frac{\alpha \beta \overline{Y^{E}}}{\lambda} \cdot\left(\frac{Z_{a, \text { eff }}}{Z_{s, \mathrm{eff}}+Z_{a, \mathrm{eff}}}\right) \cdot \bar{T}}_{\text {Part II }},
\end{aligned}
$$

where $\bar{T}=\left(2-\nu_{13} \cdot\left(C_{2} \kappa_{2} h / \tan \left(C_{2} \kappa_{2} h\right)\right)\right) /\left[\left(C_{1} \kappa_{1} R J_{0}\left(C_{1} \kappa_{1} R\right) /\right.\right.$ $\left.\left.J_{1}\left(C_{1} \kappa_{1} R\right)\right)-\nu_{13} \cdot\left(C_{2} \kappa_{2} h / \tan \left(C_{2} \kappa_{2} h\right)\right)\right]$. Part I only relates to the parameters of the PZT patch and is independent of the host structure. Part II represents the coupling between the PZT patch and the host structure. Hence, (22) is written as

$$
\bar{Y}=\overline{Y_{P}}+\overline{Y_{A}} \text {, }
$$

where $\overline{Y_{P}}$ describes the PZT contribution that is insensitive to any structural changes; it is the "passive" part; $\overline{Y_{A}}$ represents the contribution of the PZT-structure interaction and is 
sensitive to any structural changes near the PZT patch; it is the "active" part [20]. By substituting $\overline{Y^{E}}=Y^{E}(1+\eta j)$ and $\overline{\varepsilon_{33}^{T}}=\varepsilon_{33}^{T}(1-\delta j), \overline{Y_{P}}$ is expressed as

$$
\overline{Y_{P}}=G_{P}+B_{P} j
$$

where

$$
\begin{gathered}
G_{P}=\frac{(\pi R)^{2} f}{h}\left(\delta \varepsilon_{33}^{T}+\frac{\alpha \beta Y^{E}}{\lambda} \cdot \eta\right), \\
B_{P}=\frac{(\pi R)^{2} f}{h}\left(\varepsilon_{33}^{T}-\frac{\alpha \beta Y^{E}}{\lambda}\right) .
\end{gathered}
$$

The measured electrical admittance signals contain the "passive" and "active" parts. The existence of the "passive" part influences the sensitivity of the raw electrical admittance signals for SHM. Hence, if the PZT parameters are predicted, the "passive" part can be filtered out, and the "active" part can be derived from the raw electrical admittance signals:

$$
\begin{gathered}
G_{a}=G-G_{P}, \\
B_{a}=B-B_{P} .
\end{gathered}
$$

Substituting (25) and (26) into (22), the complex form of the "active" part is expressed as

$$
\overline{Y_{A}}=G_{a}+B_{a} j=\frac{\pi R^{2} j \omega}{2 h} \cdot \frac{\alpha \beta \overline{Y^{E}}}{\lambda} \cdot \frac{Z_{a, \mathrm{eff}}}{\left(Z_{s, \mathrm{eff}}+Z_{a, \mathrm{eff}}\right)} \cdot \bar{T}
$$

\section{Experimental Investigations}

In this study, a concrete beam with an embedded PZT patch during the curing age was monitored to measure the strength gain. First, an Agilent4294A precision impedance analyzer was used for collecting the electric admittance signals. By a series of computational procedures, the "active" part associated with the mechanical impedance was derived from the raw electrical admittance signal. Finally, the "active" part was utilized to monitor the strength gain of the concrete beam during the curing age. The objective of this experiment was to demonstrate the feasibility and sensitivity of utilizing the "active" part by filtering out the PZT contribution for SHM.

4.1. Experimental Setup. In the experiment, a concrete beam with dimensions of $10 \mathrm{~mm} \times 10 \mathrm{~mm} \times 50 \mathrm{~mm}$ was used for the strength gain monitoring of concrete in the early age by the proposed approach. To measure the EMI signatures during the curing age, a PZT patch having a thin epoxy adhesive that can be regarded as a waterproof layer was embedded into the concrete structure. The diameter of the PZT patch was $20 \mathrm{~mm}$, and the thickness was approximately $2 \mathrm{~mm}$. The properties of the PZT patch are listed in Table 1 . The experimental setup for collecting the electrical admittance signals is shown in Figure 6. The patch was wired to the Agilent4294A precision impedance analyzer. Then, the
TABle 1: Properties of the PZT patch.

\begin{tabular}{lc}
\hline Physical parameter & Value \\
\hline Density, $\rho\left(\mathrm{kg} / \mathrm{m}^{3}\right)$ & 7600 \\
Young's modulus, $Y^{E}\left(\mathrm{~N} / \mathrm{m}^{2}\right)$ & $7.75 \times 10^{10}$ \\
Piezoelectric strain coefficient, $d_{31}(\mathrm{~m} / \mathrm{V})$ & $120 \times 10^{-12}$ \\
Piezoelectric strain coefficient, $d_{33}(\mathrm{~m} / \mathrm{V})$ & $280 \times 10^{-12}$ \\
\hline
\end{tabular}

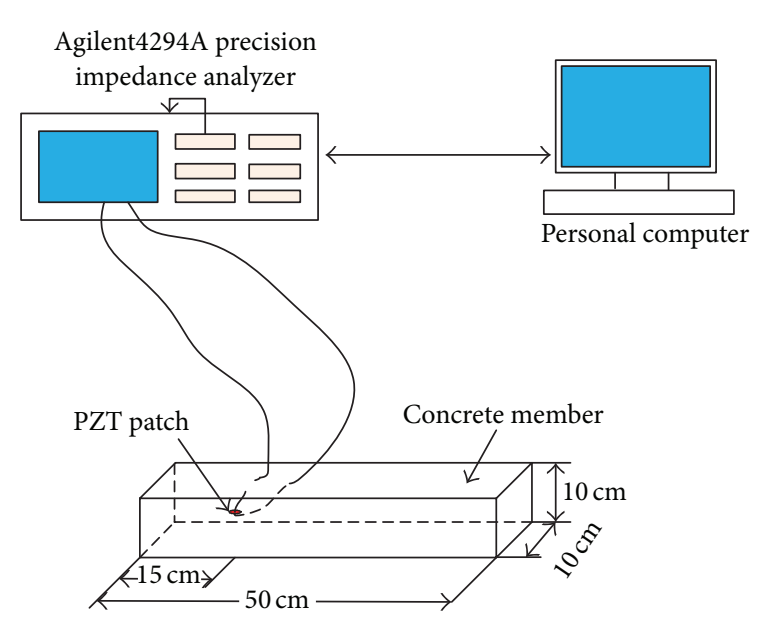

FIgURE 6: Experimental setup.

Agilent4294A precision impedance analyzer was connected to a computer via a GPIB interface.

By applying a high-frequency excitation voltage to the PZT patch with the Agilent4294A analyzer, the electromechanical admittance signature produced by the coupling vibration between the PZT patch and the host structure was measured in the frequency range of $20-200 \mathrm{kHz}$, including the real part (conductance $G$ ) and the imaginary part (susceptance $B$ ). In the monitored frequency range, 801 data points were taken with a $1-\mathrm{V}$ sweeping sine wave for the excitation. However, the measured signals are often contaminated by noise originating from the measurement system $[21,22]$. To minimize the influence of noise, eight averages of the signatures were calculated per frequency point. The impedance signature at the age of 1 day was measured and defined as the baseline, and then subsequent tests were taken at the ages of 2, 3, 4, and 7 days. By analyzing the measured electrical admittance signatures, the strength gain of the concrete beam during the curing age can be monitored by the proposed approach.

4.2. Determination of the PZT Parameters. Owing to the influence of the production process and the high temperature in the welding process between the PZT and the wires, the properties of the PZT patches provided by the manufacturer are unreliable. In the EMI technique, the "active" part of the electrical admittance signals is predicted based on the PZT parameters. Hence, more accurate PZT parameters need to be derived before extracting the "active" part from the measured raw admittance signatures. 
For a PZT patch in the free-free condition prior to its embedding into the host structure, the complex electromechanical admittance is derived as $\left(Z_{s, \text { eff }}=0\right)$

$$
\overline{Y_{\text {free }}}=G_{f}+B_{f} j=\frac{\pi R^{2} j \omega}{2 h}\left[\overline{\varepsilon_{33}^{T}}+\frac{\alpha \beta \overline{Y^{E}}}{\lambda} \cdot(T-1)\right] .
$$

By substituting $\overline{Y^{E}}=Y^{E}(1+\eta j), \overline{\varepsilon_{33}^{T}}=\varepsilon_{33}^{T}(1-\delta j), T=M+N$. $j,\left[\kappa_{1} R \cdot J_{0}\left(\kappa_{1} R\right)\right] / J_{1}\left(\kappa_{1} R\right)=r+t \cdot j,\left(\kappa_{2} h\right) / \tan \left(\kappa_{2} h\right)=r^{\prime}+t^{\prime} \cdot j$, and $\omega=2 \pi f$, we obtain

$$
\begin{gathered}
G_{f}=\frac{(\pi R)^{2} f}{h}\left[\delta \varepsilon_{33}^{T}-\frac{\alpha \beta Y^{E}}{\lambda}\{\eta(M-1)+N\}\right], \\
B_{f}=\frac{(\pi R)^{2} f}{h}\left[\varepsilon_{33}^{T}+\frac{\alpha \beta Y^{E}}{\lambda}\{(M-1)-\eta N\}\right],
\end{gathered}
$$

where

$$
\begin{gathered}
M=\frac{\left(2-v_{13} r^{\prime}\right)\left(r-v_{13} r^{\prime}\right)-v_{13} t^{\prime}\left(t-v_{13} t^{\prime}\right)}{\left(r-v_{13} r^{\prime}\right)^{2}+\left(t-v_{13} t^{\prime}\right)^{2}}, \\
N=-\frac{\left(2-v_{13} r^{\prime}\right)\left(t-v_{13} t^{\prime}\right)+v_{13} t^{\prime}\left(r-v_{13} r^{\prime}\right)}{\left(r-v_{13} r^{\prime}\right)^{2}+\left(t-v_{13} t^{\prime}\right)^{2}} .
\end{gathered}
$$

Further, for low frequencies (typically $<1 / 5$ of the first resonance frequency of the PZT), $\left[\kappa_{1} R \cdot J_{0}\left(\kappa_{1} R\right)\right] / J_{1}\left(\kappa_{1} R\right)=$ $r+t \cdot j \rightarrow 2$ and $\left(\kappa_{2} h\right) / \tan \left(\kappa_{2} h\right)=r^{\prime}+t^{\prime} j \rightarrow 1$ (i.e., $r \rightarrow 2$, $t \rightarrow 0, r^{\prime} \rightarrow 1$, and $t^{\prime} \rightarrow 0$ ); therefore, $M \rightarrow 1, N \rightarrow 0$ [13], and the quasistatic sensor approximation is expressed as [23]

$$
\begin{gathered}
G_{f, \mathrm{qs}}=\frac{(\pi R)^{2} f}{h} \cdot \delta \varepsilon_{33}^{T}, \\
B_{f, \mathrm{qs}}=\frac{(\pi R)^{2} f}{h} \cdot \varepsilon_{33}^{T} .
\end{gathered}
$$

Hence, the electrical constants $\varepsilon_{33}^{T}$ and $\delta$ can be determined by the measured electric admittance signals $G_{f, \mathrm{qs}}$ and $B_{f, \mathrm{qs}}$ for low frequencies (typically $<10 \mathrm{kHz}$ ). From this analysis, $\varepsilon_{33}^{T}$ and $\delta$ were determined to be $1.4 \times 10^{-8} \mathrm{~F} / \mathrm{m}$ and 0.0096 , respectively.

By using (29) with these PZT parameters, the electrical admittance signals of the PZT patch in the free-free condition in the frequency range of $20-220 \mathrm{kHz}$ were obtained and compared with the measured free PZT signals $(G, B)$ in Figure 7. Although the experimental and analytical free PZT signals have the good agreement in the figures, there are fundamental differences that warrant closer examination. For instance, the analytical resonance frequency is slightly higher than the experimental frequency.

In order to achieve much better agreement between the analytical and experimental signatures, the correction factors $C_{1}$ and $C_{2}$ are introduced in the terms $\left[\kappa_{1} R \cdot J_{0}\left(\kappa_{1} R\right)\right] / J_{1}\left(\kappa_{1} R\right)$ and $\left(\kappa_{2} h\right) / \tan \left(\kappa_{2} h\right)$, and these terms may be replaced by $\left[C_{1} \kappa_{1} R \cdot J_{0}\left(C_{1} \kappa_{1} R\right)\right] / J_{1}\left(C_{1} \kappa_{1} R\right)$ and $\left(C_{2} \kappa_{2} h\right) / \tan \left(C_{2} \kappa_{2} h\right)$, respectively. By trial and error, the values of the correction

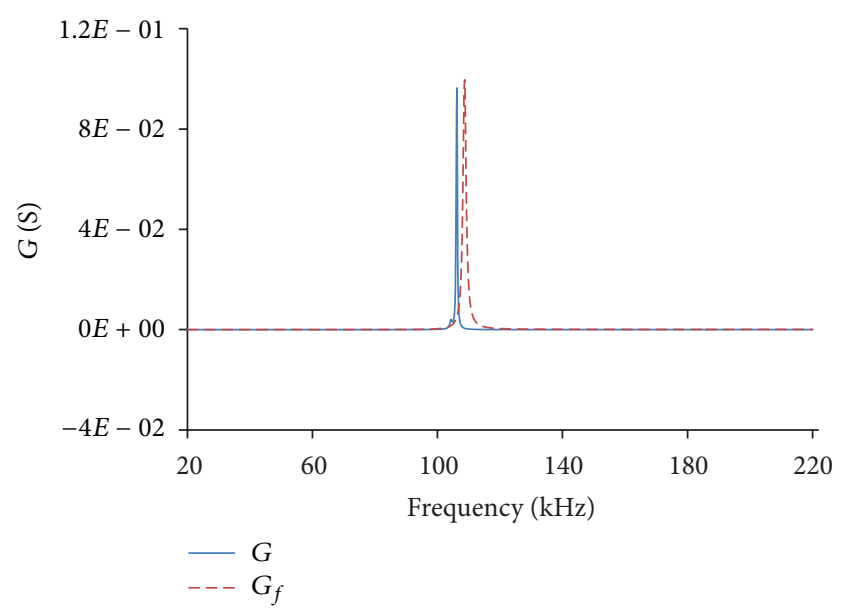

(a) Conductance

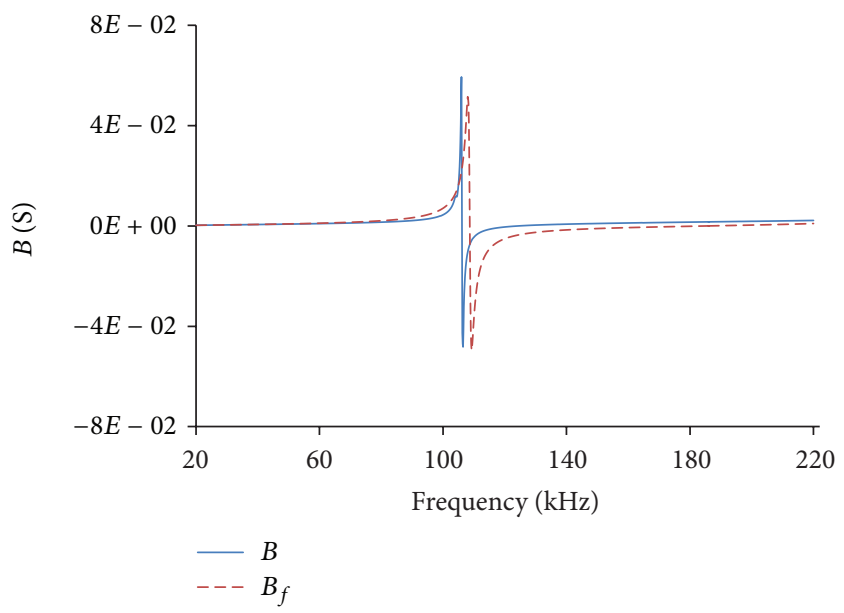

(b) Susceptance

FIgURE 7: Comparison between the experimental and theoretical signals of the PZT patch in the free condition.

factor were $C_{1}=1.023$ and $C_{2}=0.98$. Hence, very good agreement is observed between the analytical and experimental signatures. Using curve-fitting techniques, it was found that $\eta=0.021$ and $K=\left(\alpha \beta Y^{E}\right) / \lambda=7.63 \times$ $10^{-9} \mathrm{NV}^{-2}$. From these PZT parameters, the "active" part associated with the structural mechanical impedance by filtering the PZT contribution can be extracted from the measured raw admittance signatures.

4.3. Results and Analysis. As mentioned above, the PZT parameters can be accurately predicted by the measured admittance signatures of the PZT patches in the "free-free" condition. Hence, the "passive" part (PZT contribution) is filtered out, and the "active" part associated with the structural mechanical impedance can be extracted from the measured raw admittance signatures. The spectra are shown in Figure 7. With an increase in the curing time for the concrete specimen, the amplitudes of the $G_{a}$ and $B_{a}$ curves gradually diminished, and the curves shifted to the right. This indicates that the "active" part is sensitive to changes in the 


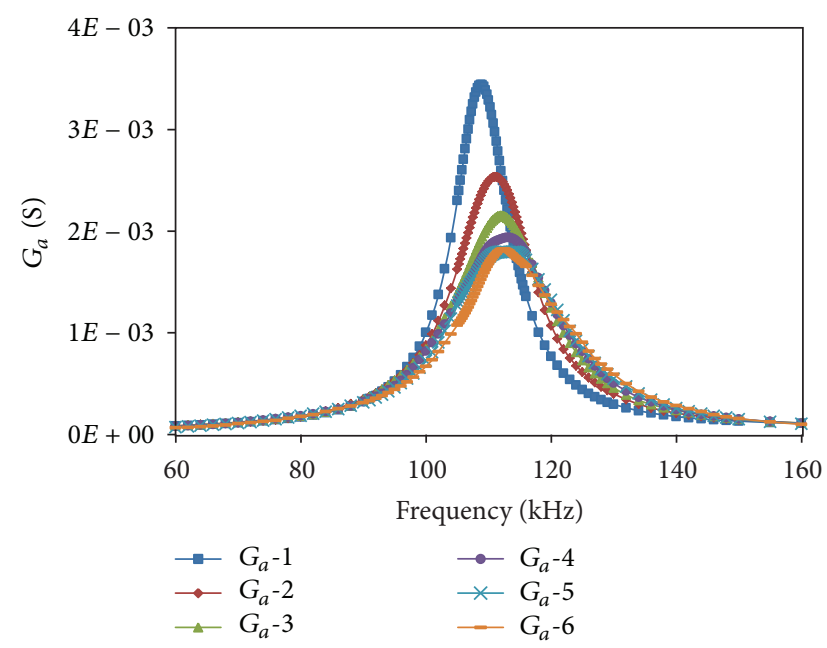

(a) Conductance

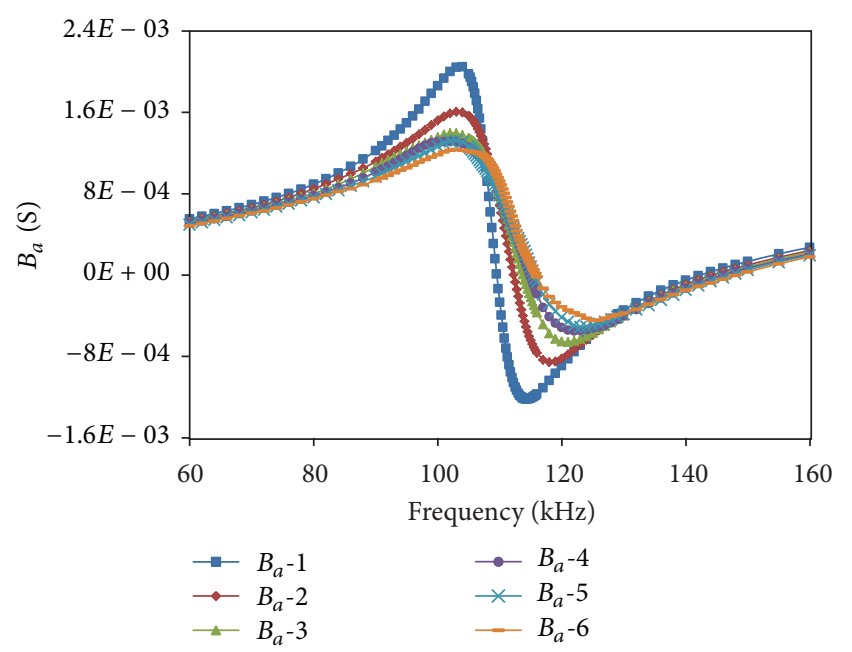

(b) Susceptance

FIGURE 8: "Active" part extracted from the measured raw admittance signatures.

inherent properties of a concrete beam with embedded PZT transducers during the curing age.

Figure 8 depicts the variations of the "active" part curve, but the extent of the changes in the inherent properties cannot be directly determined. In other words, the spectra of the "active" part only qualitatively represent the strength gain of the concrete beam during the curing age. Although the spectra are qualitatively useful, they lack any quantitative accuracy. Traditionally, statistical pattern recognition techniques have been employed to quantify the changes in the admittance signatures, such as relative deviation, root-meansquare deviation, and mean-absolute-percentage deviation [24]. In order to quantitatively analyze the strength gain for the concrete beam during the curing age, two statistical indices are defined as follows.

(1) Root-mean-square deviation (RMSD)

$$
\operatorname{RMSD}(\%)=\sqrt{\frac{\sum_{i=1}^{N}\left(y_{i}-\overline{y_{i}}\right)^{2}}{\sum_{i=1}^{N}{\overline{y_{i}}}^{2}}} \times 100 .
$$

(2) Correlation coefficient (CC)

$$
\mathrm{CC}=\frac{\left(y_{i}^{T} \cdot \bar{y}_{i}\right)^{2}}{\left|y_{i}^{T} \cdot y_{i}\right| \cdot\left|\bar{y}_{i}^{T} \cdot \bar{y}_{i}\right|},
$$

where $y_{i}$ and $y_{i}^{T}$ separately denote the vector and its transpose for the "active" part of the electrical admittance $\left(G_{a}\right.$ and $B_{a}$ ) for curing times of $2,3,4,5$, and 7 days; and $\overline{y_{i}}$ and $\bar{y}_{i}^{T}$ separately denote the vector and its transpose for the "active" part of the electrical admittance for a curing time of 1 day.

Figure 9 shows the plots of the RMSD and CC indices for the "active" part and the measured raw admittance signatures, which are calculated at various stages during the curing age with respect to the baseline signature. From Figure 8 , we find that the RMSD index increased and the
CC index decreased during the curing age for the concrete beam. These indexes qualitatively represent the variations in the inherent properties of the interior of the concrete beam.

The existing EMI techniques only depend on the real part of the measured electrical signatures, but the information in the imaginary part is lost. By eliminating the "passive" part, the admittance signatures are more sensitive to any structural changes than the raw signatures. By comparing the "active" part and the measured raw admittance signatures, the indices of the real part are similar, whereas the indices of the imaginary part have a large difference. Further, the indices of the imaginary component are similar to the indices of the real component in the "active" admittance signatures. This indicates that utilization of the imaginary component for damage identification is feasible. The experimental results demonstrate that the "active" part of the admittance signatures is more sensitive than the raw admittance signatures for SHM. Furthermore, the imaginary component can also be used for SHM.

\section{Conclusions}

In this paper, a three-dimensional effective electromechanical impedance model for an embedded PZT transducer is proposed. Based on the concept of effective impedance, the 3D coupled electromechanical admittance equation is derived. By rearranging the various terms, the admittance equation is separated into an "active" part and a "passive" part. Because the "active" part is sensitive to any structural changes near the PZT patch, it is feasible to use the "active" part for SHM. Then, the proposed method is used to monitor the strength gain of a concrete beam with embedded PZT transducers during the curing age. The results show that the "active" part of the admittance signatures is more sensitive to any structural damage than the raw admittance signatures, which demonstrates the validity of the proposed method. 

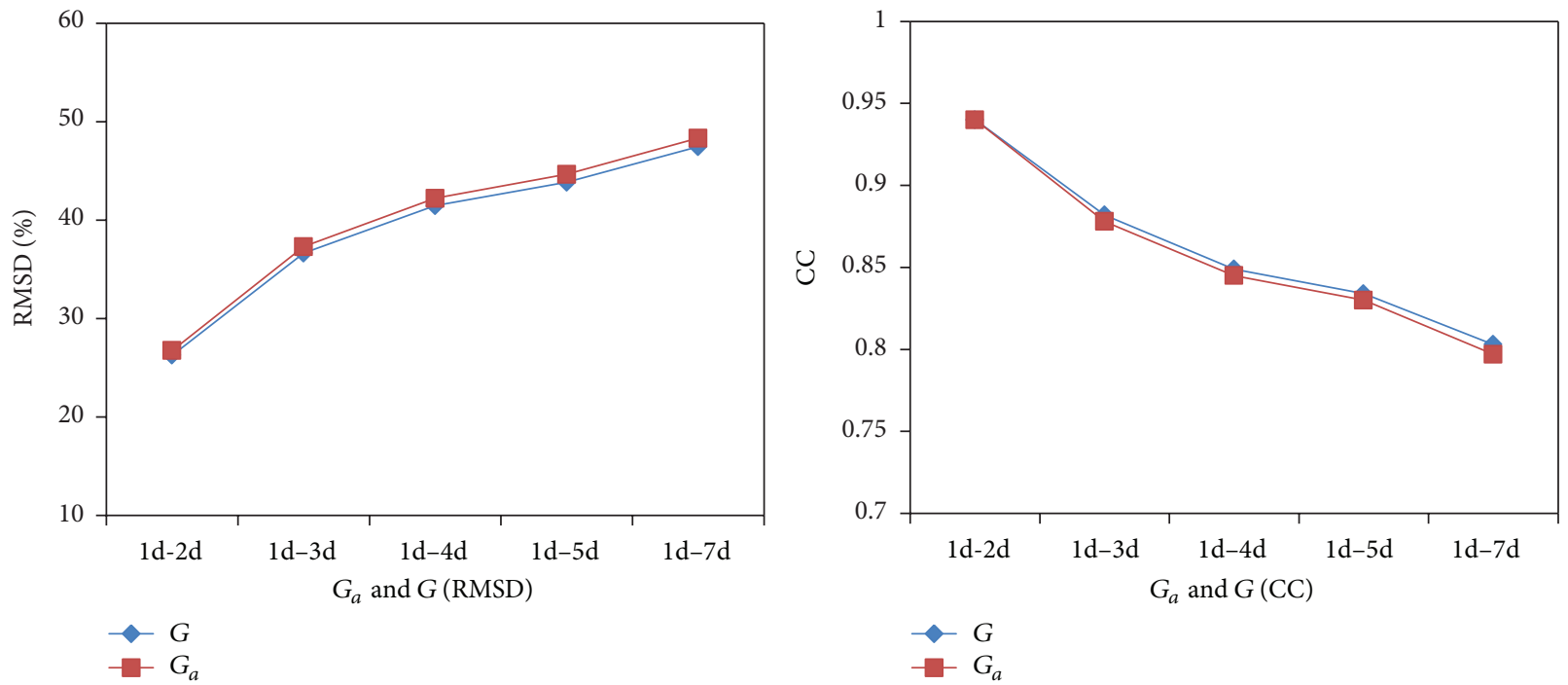

(a) $G_{a}$ and $G$
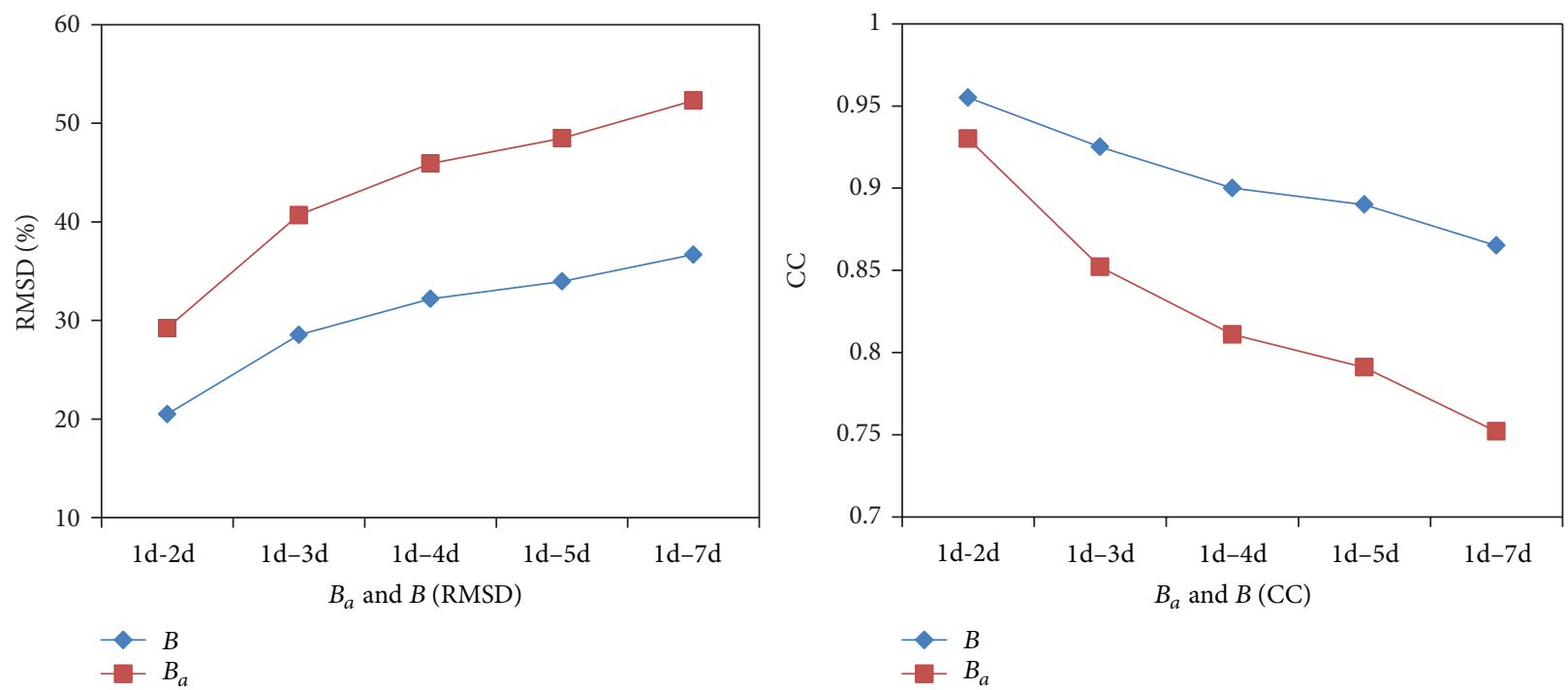

(b) $B_{a}$ and $B$

FIGURE 9: Comparison between the "active" part and the measured raw admittance signatures.

\section{Acknowledgments}

This work was supported by the Sate Key Development Program for Basic Research of China with Grant no. 2013CB035906 and Grants from the National Natural Science Foundation of China $(51121005,51078060)$. The authors gratefully acknowledge the support from the Education Department of Liaoning Province with Grant no. L2012010.

\section{References}

[1] T. Yi, H. Li, and M. Gu, "Optimal sensor placement for health monitoring of high-rise structure based on genetic algorithm," Mathematical Problems in Engineering, vol. 2011, Article ID 395101, 12 pages, 2011.
[2] T. H. Yi and H. N. Li, "Methodology development in sensor placement for health monitoring of civil infrastructures," International Journal of Distributed Sensor Networks, vol. 2012, Article ID 612726, 11 pages, 2012.

[3] I. Chopra, "Review of state of art of smart structures and integrated systems," AIAA Journal, vol. 20, no. 11, pp. 2145-2187, 2002.

[4] S. Yan, N. Z. Zhao, Q. J. Wang, and G. B. Song, "Dynamic mechanical model of surface-bonded PZT actuator: theory and experiment," Applied Mechanics and Materials, vol.303-306, pp. 1732-1735, 2013.

[5] K. Nguyen, J. Park, and J. Kim, "Imote2-based multi-channel wireless impedance sensor nodes for local SHM of structural connections," in Sensors and Smart Structures Technologies for Civil, Mechanical, and Aerospace Systems, vol. 7981 of Proceedings of SPIE, pp. 79811P-79811P-11, San Diego, Calif, USA, March 2011. 
[6] J. T. Kim, K. D. Nguyen, and J. H. Park, "Wireless impedance sensor node and interface washer for damage monitoring in structural connections," Advances in Structural Engineering, vol. 15, no. 6, pp. 871-885, 2012.

[7] K. D. Nguyen, P. Y. Lee, and J. T. Kim, "Smart PZT-interface for SHM in tendon-anchorage of cable-stayed bridge," in Sensors and Smart Structures Technologies for Civil, Mechanical and Aerospace System, vol. 8345 of Proceedings of SPIE, pp. 834519834519-11, San Diego, Calif, USA, March 2012.

[8] Z. A. Chaudhry, T. Joseph, F. P. Sun, and C. A. Rogers, "Local-area health monitoring of aircraft via piezoelectric actuator/sensor patches," in Smart Structures and Materials 1995: Smart Structures and Integrated Systems, vol. 2443 of Proceedings of SPIE, pp. 268-276, San Diego, Calif, USA, March 1995.

[9] J. W. Ayres, F. Lalande, Z. Chaudhry, and C. A. Rogers, "Qualitative impedance-based health monitoring of civil infrastructures," Smart Materials and Structures, vol. 7, no. 5, pp. 599605, 1998.

[10] G. Song, H. Gu, Y. L. Mo, T. T. C. Hsu, and H. Dhonde, "Concrete structural health monitoring using embedded piezoceramic transducers," Smart Materials and Structures, vol. 16, no. 4, pp. 959-968, 2007.

[11] G. Park, H. H. Cudney, and D. J. Inman, "Feasibility of using impedance-based damage assessment for pipeline structures," Earthquake Engineering and Structural Dynamics, vol. 30, no. 10, pp. 1463-1474, 2001.

[12] G. Park, H. Sohn, C. R. Farrar, and D. J. Inman, "Overview of piezoelectric impedance-based health monitoring and path forward," Shock and Vibration Digest, vol. 35, no. 6, pp. 451-463, 2003.

[13] C. Liang, F. P. Sun, and C. A. Rogers, "Coupled electromechanical analysis of adaptive material systemsdetermination of the actuator power consumption and system energy transfer," Journal of Intelligent Material Systems and Structures, vol. 5, no. 1, pp. 12-20, 1994.

[14] S. Zhou, C. Liang, and C. A. Rogers, "An impedance-based system modeling approach for induced strain actuator-driven structures," Journal of Vibration and Acoustics, vol. 118, no. 3, pp. 323-331, 1996.

[15] S. Bhalla and C. K. Soh, "Structural health monitoring by piezoimpedance transducers. I: modeling," Journal of Aerospace Engineering, vol. 17, no. 4, pp. 154-165, 2004.

[16] E. F. Crawley and J. de Luis, "Use of piezoelectric actuators as elements of intelligent structures," AIAA Journal, vol. 25, no. 10, pp. 1373-1385, 1987.

[17] S. Y. Lin, "Coupled vibration analysis of piezoelectric ceramic disk resonators," Journal of Sound and Vibration, vol. 218, no. 2, pp. 205-217, 1998.

[18] IEEE, “IEEE standard on piezoelectricity," IEEE 176-1987, 1988.

[19] A. H. Meitzler, H. M. O’Bryan, and H. F. Tiersten, "Definition and measurement of radial mode coupling factors in piezoelectric ceramic materials with large variations in Poisson's ratio," IEEE Transactions on Sonics and Ultrasonics, vol. 20, no. 3, pp. 233-239, 1973.

[20] S. Bhalla and C. K. Soh, "Structural health monitoring by piezoimpedance transducers. II: applications," Journal of Aerospace Engineering, vol. 17, no. 4, pp. 166-175, 2004.

[21] T. H. Yi, H. N. Li, and X. Y. Zhao, "Noise smoothing for structural vibration test signals using an improved wavelet thresholding technique," Sensors, vol. 12, no. 8, pp. 11205-11220, 2012.
[22] T. H. Yi, H. N. Li, and H. M. Sun, "Multi-stage structural damage diagnosis method based on "energy-damage" theory," Smart Structures and Systems, vol. 12, no. 3-4, pp. 345-361, 2013.

[23] V. Giurgiutiu and A. N. Zagrai, "Embedded self-sensing piezoelectric active sensors for on-line structural identification," Journal of Vibration and Acoustics, vol. 124, no. 1, pp. 116-125, 2001.

[24] K. K.-H. Tseng and A. S. K. Naidu, "Non-parametric damage detection and characterization using smart piezoceramic material," Smart Materials and Structures, vol. 11, no. 3, pp. 317-329, 2002. 


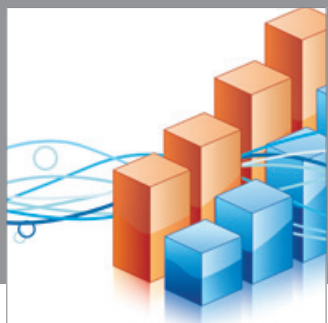

Advances in

Operations Research

mansans

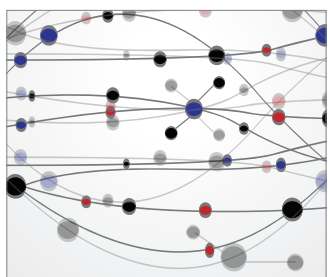

The Scientific World Journal
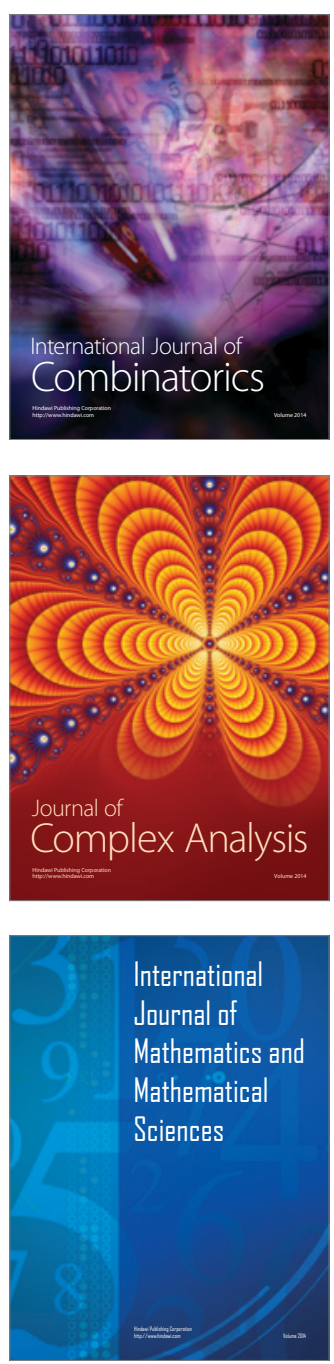
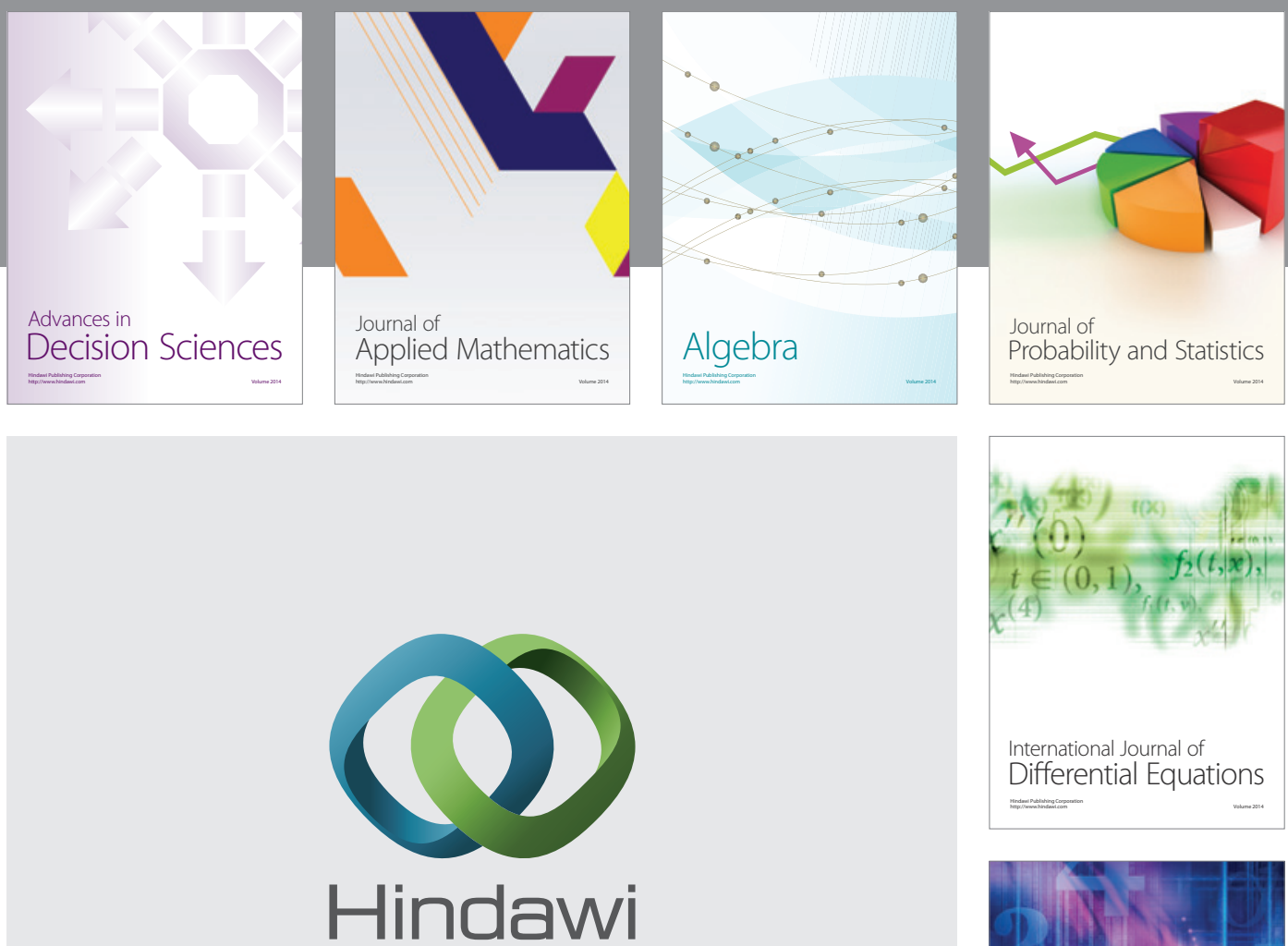

Submit your manuscripts at http://www.hindawi.com
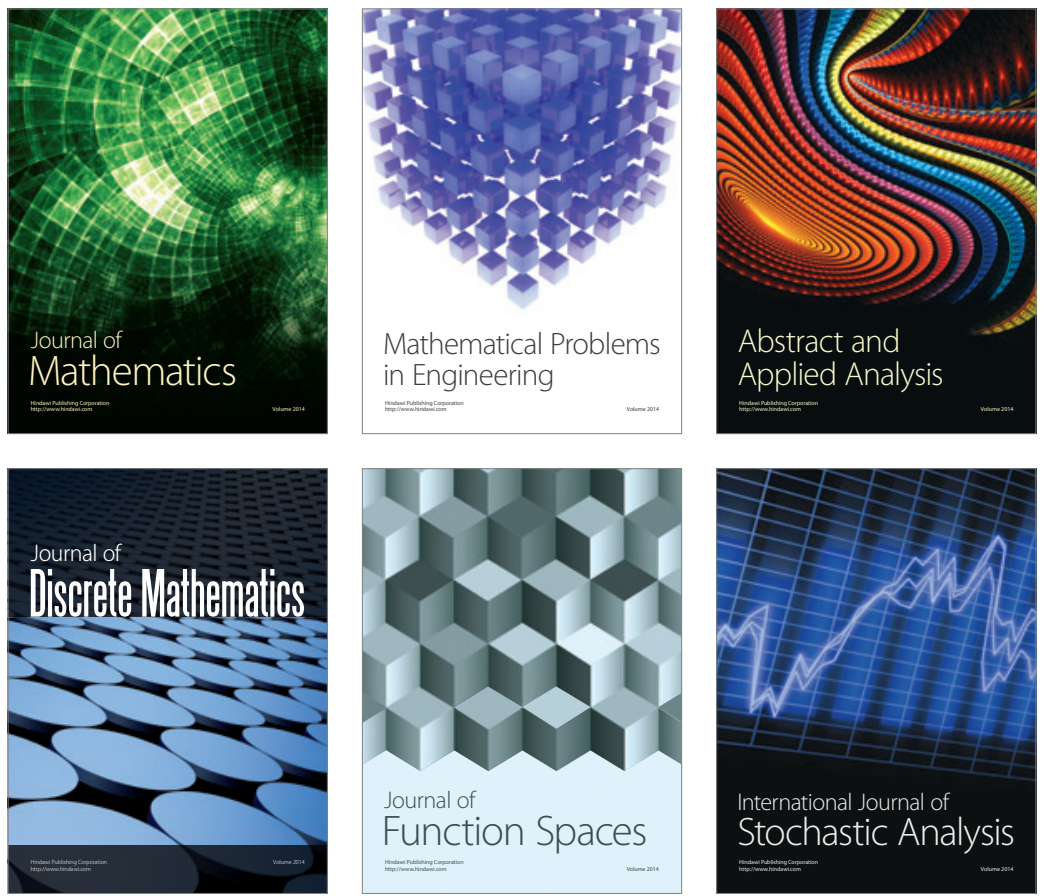

Journal of

Function Spaces

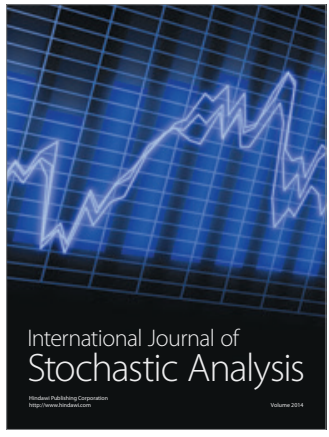

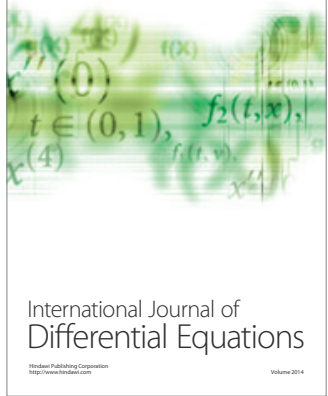
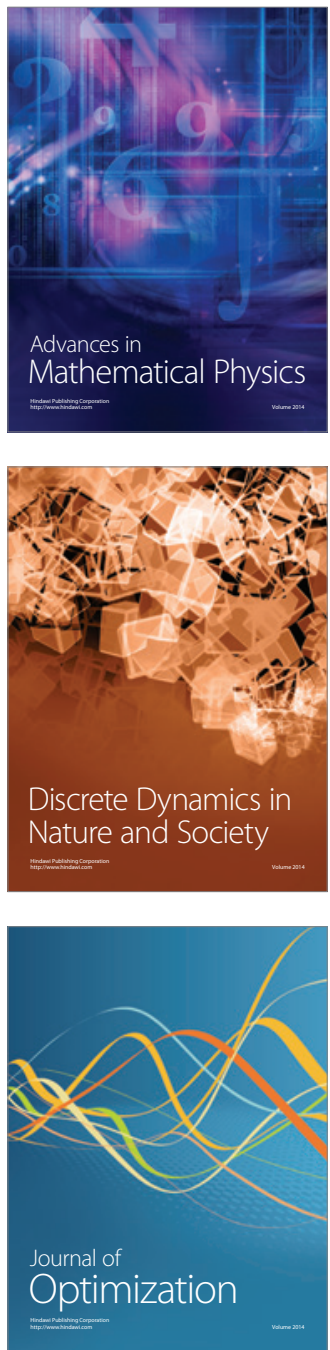